\title{
Publisher Correction: Attractor dynamics gate cortical information flow during decision-making
}

Arseny Finkelstein (D), Lorenzo Fontolan (1), Michael N. Economo, Nuo Li, Sandro Romani (i) and Karel Svoboda (i)

Correction to: Nature Neuroscience https://doi.org/10.1038/s41593-021-00840-6, published online 19 April 2021.

In the version of this article initially published, errors occurred in the Fig. 4a legend; in Equations 6 and 10; in the Methods text; and in the Extended Data Fig. 7a legend.

Equation 6 should have read:

$$
\Delta \overline{\mathbf{r}}=\overline{\mathbf{r}}_{\text {Delay }}-\overline{\mathbf{r}}_{\text {Pre-sample }}
$$

Equation 10 should have read:

$$
\tau \dot{\mathbf{x}}(t)=-\mathbf{x}(t)+W_{\text {rec }} \mathbf{r}(t)+\mathbf{w}_{\text {ramp }} I_{\text {ramp }}(t)+\mathbf{w}_{\text {stim }} I_{\text {stim }}^{K}(t) \mathbf{w}_{\text {cue }} I_{\text {cue }}(t)+\sigma_{\eta} \boldsymbol{\eta}(t)
$$

In the Methods, in the paragraph following Equation 11, in the in the sentence beginning "The weight vectors were..." a spurious curly bracket appeared; and in the third paragraph of the section "Network training and testing" a close-parenthesis was missing from the sentence beginning "We excluded aberrant trials...."

In the legends of Fig. 4a and Extended Data Fig. 7a, the sentences beginning "A linear ramping input..." should have described the linear ramping input as purple rather than black.

The errors have been corrected in the PDF and HTML versions of this article.

Published online: 19 May 2021

https://doi.org/10.1038/s41593-021-00869-7

(c) The Author(s), under exclusive licence to Springer Nature America, Inc. 2021

\section{Publisher Correction: Closing the gate to distractors during decision-making}

Edmund Chong and Athena Akrami (D)

Correction to: Nature Neuroscience https://doi.org/10.1038/s41593-021-00833-5, published online 19 April 2021.

In the version of this article initially published, spelling errors occurred in the figure labels. The errors have been corrected in the PDF and HTML versions of this article.

Published online: 17 May 2021

https://doi.org/10.1038/s41593-021-00870-0

(๑) Springer Nature America, Inc. 2021 